\title{
A hybrid mixed double-sided incremental forming method for forming Ti6Al4V alloy
} \author{
aStefania Bruschi (2), cJian Cao (1)* \\ a Department of Industrial Engineering, University of Padova, Padova, Italy \\ bDepartment of Mechanical Engineering (EPSEVG), Tecnofab group, Universitat Politècnica de Catalunya, Spain \\ cDepartment of Mechanical Engineering, Northwestern University, Evanston, IL, USA \\ \#Equal contributions
}

aBeatrice Valoppi", bAntonio J. Sánchez Egea\#, cZixuan Zhang”, bHernán A. González Rojas, aAndrea Ghiotti,

Incremental forming can be an alternative manufacturing means in producing biomedical parts characterized by the need of patient-specific geometry. A novel hybrid dieless sheet metal forming process, Electrically-assisted Mixed Double-Sided Incremental Forming (E-MDSIF), is proposed to manufacture difficult-to-form Ti6Al4V sheets. E-MDSIF is a spark-free technique shown to increase the formability and the geometric accuracy while decreasing the forming force. The effects of the electrical process on microstructure, micro-hardness and surface roughness of the formed parts are investigated. Additionally, comparisons between E-MDSIF and the conventional E-ISF processes are illustrated based on fundamental mechanics. Limitations and potential applications are presented.

Incremental sheet forming, Titanium, Electrically-assisted

\section{Introduction}

Titanium and its alloys have been widely used for many different applications in the aerospace and biomedical industries due to their high strength-to-weight ratio, elevated corrosion resistance and excellent biocompatibility. In particular, Ti6Al4V is the most used titanium alloy for the production of biomedical implants, which can be often manufactured through machining and additive manufacturing technologies [1]. An alternative route to produce high surface-to-thickness products is represented by Incremental Sheet Forming (ISF) processes [2]. In ISF, the periphery of the sheet metal is clamped, while the material is locally deformed by one, i.e., Single Point Incremental Forming (SPIF), or more stylus tools that move along pre-defined toolpaths. Therefore, because of their dieless nature and high flexibility, these techniques are most suitable when low-volume customized sheet metal parts, as the case of biomedical implants, have to be manufactured [3].

To improve the final geometric accuracy achievable with SPIF, different variants of the ISF process have emerged, such as MultiPass Incremental Forming covering the entire [4] or partial [5] forming areas repeatability, and Double-Sided Incremental Forming (DSIF) [6]. Furthermore, significant improvements in geometric accuracy have been reached employing a new toolpath approach, named Accumulative Double-Sided Incremental Forming (ADSIF) [7]. Unlike the conventional out-to-in and topto-bottom toolpath used in DSIF, the ADSIF strategy allows the forming tools deforming the material from the inner to the outer circumference at a given horizontal plane. Zhang et al. [8] proposed a Mixed Double-Sided Incremental Forming (MDSIF) process in which the part is first formed by ADSIF and then finetuned by DSIF. With this process, a better geometric accuracy and shorter forming time were reported.

One challenge in all the above incremental forming techniques is forming workpieces made of lightweight but difficult-to-form materials [3], such as Ti6Al4V. Because of its limited formability at room temperature [9], a possible solution to form Ti6Al4V sheets using ISF processes is to increase the forming temperature. According to the literature, many techniques, based on different heating methods, such as conduction, radiation, friction and electricity [3], have been proposed. Among them, the Electrically-assisted Incremental Sheet Forming process (E-ISF) has the great potential because it is the most flexible and costeffective in terms of equipment and temperature control. Nevertheless, E-ISF process shows two main drawbacks, namely the limited geometric accuracy of the formed part and the poor final surface quality [3]. The rough surface from E-ISF may be a challenge for all aesthetic purposes, but it is advantageous for those biomedical applications whose surface plays a key role in the implant-bone interaction [10]. Indeed, it has been demonstrated that high surface roughness enhances the osseointegration process [11]. Therefore, for the production of a part made in Ti6Al4V and characterized by a double curvature used in the hip replacement, E-ISF process allows combining the deformation process and the surface modification aimed at increasing the surface roughness. Although Electrically-assisted DSIF (E-DSIF) has been realized to overcome the limited achievable geometric accuracy [12], sparks, which causes damage to the sheet and the tool, happened during the forming process due to lack of contact between the bottom tool and the sheet metal. Losing contact has been frequently observed in DSIF, thus complicated equipment and compensation algorithm have been realized to address this issue [13].

To overcome the issue of the limited geometric accuracy inherent to the E-ISF process and the spark phenomenon in EDSIF, the paper proposes two spark-free approaches based on the ADSIF technology, originated in Malhotra et al. [7], combined with the tool-gap compensation algorithm developed to avoid the loss of contact in DSIF [8]. Note that in ADSIF, the tools always form the virgin material. Therefore, the contact between the tools and the sheet is guaranteed, which eliminates the spark problem. Specifically, the two proposed methods are the Electricallyassisted Accumulative Double-Sided Incremental Forming (E- 
ADSIF) and the Electrically-assisted Mixed Double-Sided Incremental Forming (E-MDSIF), respectively. In this paper, the two forming methodologies will be elaborated in Section 2, followed by the results on the effects of current density and its associated Joule heating effect on geometric accuracy, forming force and material microstructure in Section 3. Finally, discussions on the results, its advancement and limitations, and potential applications will be presented in Section 4 .

\section{Methodology}

The material used in the current study is $0.5 \mathrm{~mm}$ thick Ti6Al4V sheet in annealed conditions. Its microstructure in the asreceived condition consists of equiaxed $\alpha+\beta$ [14], as shown in Fig. 1(a).
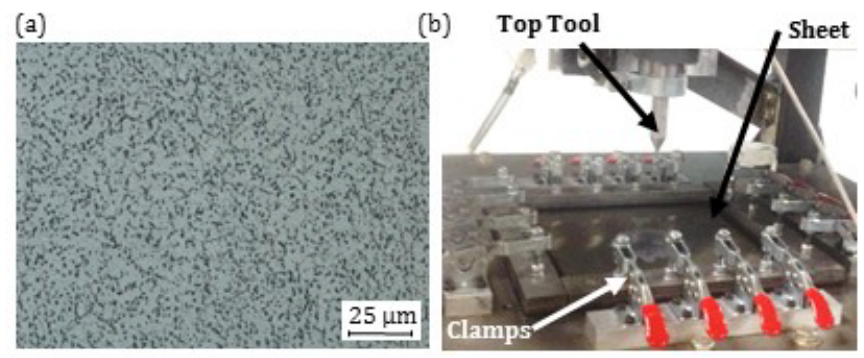

Figure 1. (a) Microstructure of Ti6Al4V in the as-received condition. (b) Details of DSIF clamping system and tool setups.

The experimental tests were performed on the custom-built DSIF machine housed at Northwestern University. The Ti6Al4V sheets $(320 \mathrm{~mm} \times 320 \mathrm{~mm})$ were isolated at the edges with temperature-resistant insulator tape $\left(-18^{\circ} \mathrm{C}\right.$ to $\left.105^{\circ} \mathrm{C}\right)$, and then clamped by using a proper clamping system as shown in Fig. 1(b). Two hemispherical tools made of tool steel (60 HRC) with diameter of $5 \mathrm{~mm}$ were used as "Top tool" (Fig. 1(b)) and "Bottom tool" (not shown in the figure, blocked by the sheet metal). The D and S parameters, which define the position of the bottom tool with respect to the top tool during the forming process [8] were set at 2.5 and $0.43 \mathrm{~mm}$, respectively, as seen in Fig. 2. The tools moved along spiral toolpaths with a constant incremental depth of $0.1 \mathrm{~mm}$. The tool speed was set as $600 \mathrm{~mm} / \mathrm{min}$. The forming forces were recorded by two multi-axis load cells.

To electrically assist the forming process, a continuous current power supplier (Dynatronix, model LFP12-300) was integrated to the experimental set-up, as depicted in Fig. 2, creating a closed circuit. In all the experiments, the electricity was applied after the contacts between the sheet and the forming tools were made initially in order to avoid electric arcs that could damage both the sample and the tools. Polymeric matrixes were utilized to isolate the tools from the DSIF machine.

To analyse how the electricity affects the material formability and geometric accuracy, the experiments were first carried out without electricity at room temperature ("Base line"), and then with different current intensities, spanning from $40 \mathrm{~A}$ to $120 \mathrm{~A}$ in the E-ADSIF cases. The E-MDSIF process was performed using the best current value obtained from the E-ADSIF cases. The results from E-ADSIF and E-MDSIF using the same current value were then compared in terms of geometric accuracy and postdeformation material properties. To reduce the friction during the forming phase, conductive $\mathrm{MoS}_{2}$ powder was used as lubricant at the tool-sheet interfaces.

The surface temperature was measured using an infrared (IR) camera (Micro-Epsilon, model TIM160 Imager) with a temperature range from $-20{ }^{\circ} \mathrm{C}$ to $900{ }^{\circ} \mathrm{C}$. The Ti6Al4V emissivity in the testing conditions was set at 0.75 after a calibration process, which consisted in the comparison between the temperature values recorded by the IR camera and the ones resulted from a thermocouple placed on the sheet.

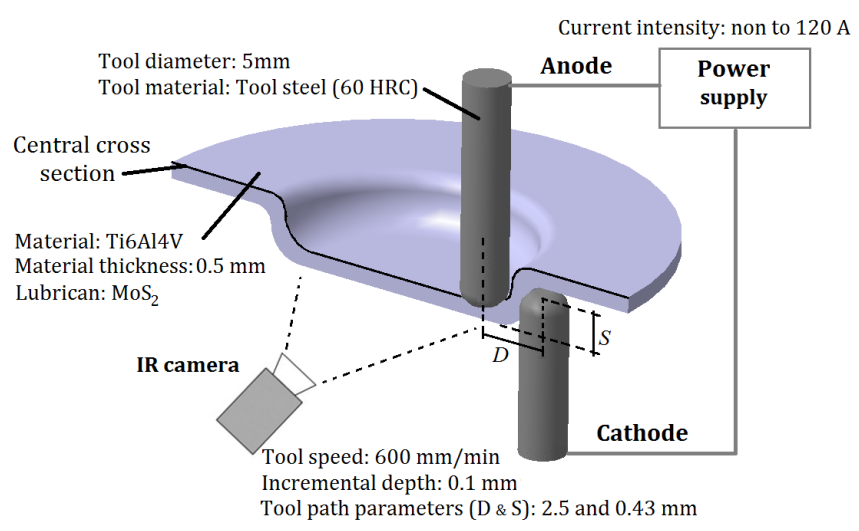

Figure 2. Sketch of the experimental set-up used for E-ISF process and working parameters.

The part geometry to be tested in this work is a part with a total depth of $20 \mathrm{~mm}$ and a double curvature with wall angles of $20^{\circ}$ and $50^{\circ}$, respectively, as shown in Fig. 3 . The double-curvature shape was chosen because it is characteristic of the acetabular cups used in the hip replacement.

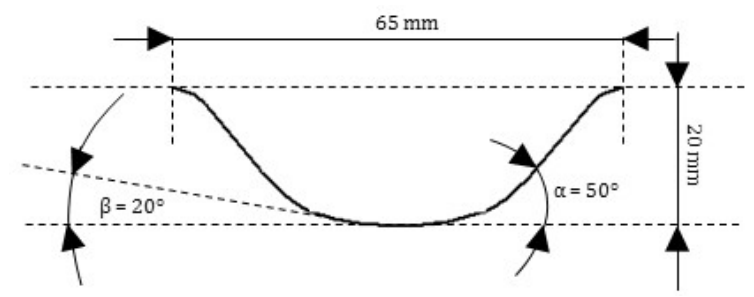

Figure 3. CAD drawing of the part examined (desired geometry).

The geometric accuracy was evaluated by comparing the resulting geometries of the formed parts, measured with a noncontact 3D laser scanner (Minolta, model VIVID 900), with respect to the desired one. After being scanned, the formed parts were cut at the central cross section (Fig. 2) to allow more characterization work, namely the surface roughness, the microstructures and the micro-hardness. The average surface roughness of the as-received material and the formed parts (both internal and external surfaces), $R_{a}$, was measured with an optical surface profiler (Alicona, InfiniteFocus). The micro-hardness tests were performed on a micro-durometer (Struers, model Duramin 5) and the reported results are the average values acquired along the thickness and far enough from the edges of the samples. The microstructures of the specimens revealed using the Kroll's reagent were analysed along the thickness with an optical microscope (Nikon, model Optiphot-10). They were evaluated to examine any effect on the microstructural features that could be associated to the applied electric configurations.

\section{Results and discussion}

\subsection{Geometric accuracy}

The geometries of the formed parts are compared with the desired one in Fig. 4, while Table 1 reports the values of the parameters used to characterize the geometric accuracy. It was evaluated considering the resulting two wall angles, the achieved depth and the fracture occurrence. 
First, among all the 'E-ADSIF' cases, the forming heights were increased from the base line case $(0 \mathrm{~A})$, to $40 \mathrm{~A}$ and then $50 \mathrm{~A}$ cases, but decreased in the $100 \mathrm{~A}$ and $120 \mathrm{~A}$ cases. The progressive wear of the tools, linked to the rise of temperature resulting from the increase of the current intensity, develops a negative effect on the material. Figure 4 shows that the best geometrical approximation compared to the desired shape was achieved when a current intensity of $50 \mathrm{~A}$ was applied.

According to Fig. 4 and Table 1, the E-MDSIF outperforms all the E-ADSIF cases significantly, reaching the highest values of total depth and wall angles. This could be attributed to the fact that the material is first moved from the inner to the outer circumference in E-ADSIF and then redistributed during E-DSIF.

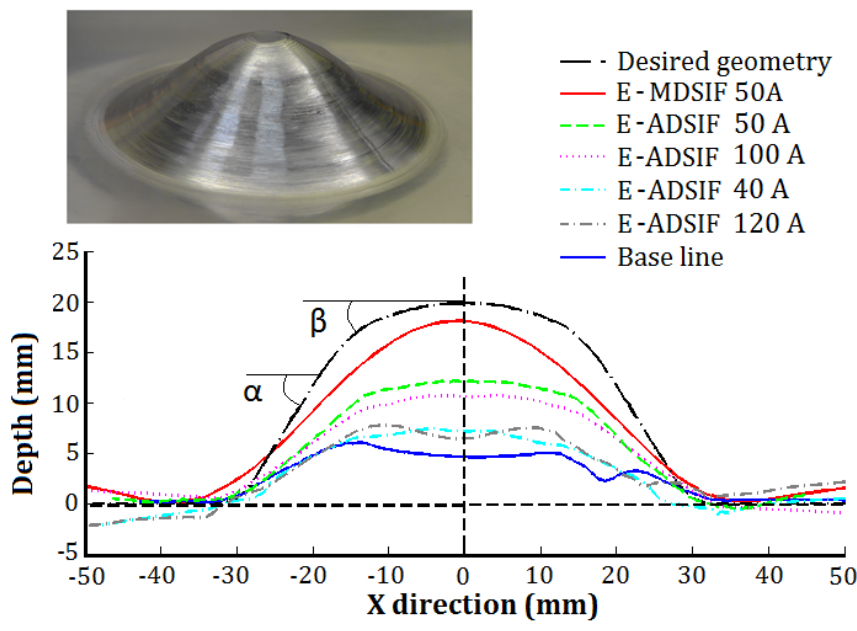

Figure 4. Formed part geometry for each configuration.

Table 1. Geometric accuracy for each configuration.

\begin{tabular}{ccccccc}
\hline Part & $\begin{array}{c}\mathbf{I} \\
{[\mathbf{A}]}\end{array}$ & $\begin{array}{c}\text { Depth } \\
{[\mathbf{m m}]}\end{array}$ & $\begin{array}{c}\boldsymbol{\alpha} \\
{\left[{ }^{\mathbf{0}}\right]}\end{array}$ & $\begin{array}{c}\boldsymbol{\beta} \\
{\left[{ }^{\mathbf{0}} \mathbf{]}\right.}\end{array}$ & Fracture \\
\hline \hline Desired & - & 20 & & 50 & 20 & - \\
\hline Base line & - & 4.58 & 20.45 & - & Yes \\
\hline & 40 & 7.20 & 24.44 & 3.27 & Yes \\
E-ADSIF & 50 & 12.02 & 35.88 & 10.84 & No \\
& 60 & 7.88 & 19.13 & 2.73 & No \\
& 100 & 10.58 & 29,48 & 8.33 & No \\
\hline E-MDSIF & 120 & 6.48 & 24.72 & - & Yes \\
\hline
\end{tabular}

\subsection{Forming forces and temperatures}

The forming forces of the top and bottom tools along the $\mathrm{x}, \mathrm{y}$ and $\mathrm{z}$ directions, named $\mathrm{F}_{\mathrm{x}}, \mathrm{F}_{\mathrm{y}}$ and $\mathrm{F}_{\mathrm{z}}$, respectively, were elaborated to calculate an average total forming force for each tool by using the following equation:

$$
F_{T}=\frac{1}{T} \int_{0}^{T} \sqrt{F_{x}^{2}+F_{y}{ }^{2}+F_{z}^{2}} \cdot d t
$$

where $F_{T}$ is the average total forming force and $\mathrm{T}$ is the period of time of the forming process.

In order to estimate how the temperature, generated by the Joule effect, influences the forming forces, a normalized forming force, $F_{N}$, is calculated by dividing the average forming forces, $F_{T}$, of the E-ADSIF with the ones acquired during the "base line" experiment. The values of $F_{N}$ for both the bottom and the top tool are reported in Fig. 5. These results indicate that the E-ADSIF decreases the forming forces needed. This force reduction is attributed to the increase of the forming temperature, which results from the application of electricity. The effect of temperature on flow stress reduction of Ti6Al4V in a wide range of temperatures was reported in [1]. The maximum decrease of forming forces was calculated when a current intensity of $100 \mathrm{~A}$ was applied and was found equal to $14.5 \%$, while the minimum, namely 3\%, was observed at $40 \mathrm{~A}$. Nevertheless, a decrease of about $8 \%$ was found when the process was assisted with $120 \mathrm{~A}$. Therefore, it seems that above $100 \mathrm{~A}$ the temperature becomes a drawback for further reduction of forming forces. This could be explained by the significant wear of the tools observed due to the resulting high temperature when higher current intensities were applied. Material oxidation at that temperature value can also be a factor in the increase of forming force.

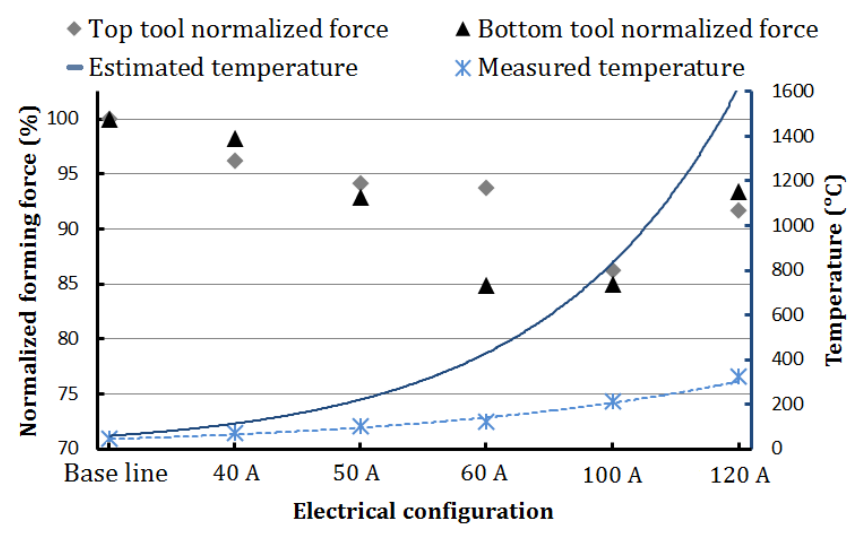

Figure 5. Top and bottom tool normalized forces and associated measured and estimated temperatures.

As the localized heating zone was blocked by the tools in the view of the IR camera, the measured temperatures, reported in Fig. 5, refer to the surroundings of the tool-sheet contact area. Therefore, to estimate the temperature right under the sheet-tool contact area, an approach similar to the one used in [15] was applied. This instantaneous temperature was roughly estimated assuming an adiabatic condition and the cross section of the contact area circular with a radius of $0.25 \mathrm{~mm}$, as resulted from experimental observations. Therefore, the instantaneous rise of temperature by the Joule effect at the sheet-tool contact is estimated to be:

$$
\Delta T=(c \cdot \rho)^{-1} \cdot \gamma \cdot\left(\frac{I}{\pi \cdot r^{2}}\right)^{2} \cdot t
$$

where I is the current intensity, $\mathrm{r}$ the radius of the cross sectional area, $\mathrm{t}$ the time $(0.05 \mathrm{~s}$, estimated from the applied tool speed), $\gamma$ $\left(1.78 \cdot 10^{-7} \Omega \mathrm{m}\right), \mathrm{C}\left(526 \mathrm{~J} /\left(\mathrm{kg} \cdot{ }^{\circ} \mathrm{C}\right)\right)$ and $\rho\left(4.43 \cdot 10^{3} \mathrm{~kg} / \mathrm{m}^{3}\right)$ are the electrical resistivity, specific heat and density, respectively. Observing the estimated and the measured thermal values reported in Fig. 5, the estimated temperature at the tool-sheet contact area is much higher than the measured value of the surrounding, which is in agreement with [16]. Note that heat loss was not considered in the estimated temperature.

\subsection{Metallography}

The results of the surface analysis, microstructural observations and micro-hardness measurements are shown in Fig. 6, as a function of the current intensity and forming strategy. The average values of the surface roughness $\left(R_{a}\right)$ and microhardness, along with their standard deviations, are calculated from at least 15 measurements along the specimen.

Figure 6 (a) shows that even the sole ADSIF process ("Base line") allows increasing the surface roughness compared to one of the as-received sheet, but the application of the electricity makes this effect more significant, especially in case of the internal surface. Indeed, a higher current intensity triggers the oxidization process on both internal and external surfaces, but the higher forming pressure applied by the top tool increases the mechanical 
effect on the internal surface, i.e., removing the oxide layer, and at the same time increasing the average roughness [17]. Concerning the effect of various forming strategies, the E-MDSIF, namely EADSIF followed by E-DSIF in the second step, resulted in the highest values of the surface roughness, thus making the E-MDSIF strategy suitable for the production of biomedical applications.

The micrographs reported in Fig. 6(b) show the same equiaxed $\alpha+\beta$ microstructure of the as-received sheet [14], proving that, for current intensities lower than $100 \mathrm{~A}$, the forming temperature did not exceed the $\beta$-transus temperature (approx. $995^{\circ} \mathrm{C}$ ), as it was predicted considering the estimated temperatures reported in Fig. 5. The "Base line" specimen shows several cracks along the profile of the internal surface orientated from the top to the bottom (Fig. 6(b)-I). These cracks were found in the middle part of the specimen, where the wall angle is $50^{\circ}$. In this particular region, the material, hardened by the previous plastic deformation, experiences a stretching mechanism. This stretch along with the reduction of the sheet thickness could be one of the reasons for the onset of these cracks. On the contrary, no cracks were found in the electrically-formed specimens because the thermal contribution affects the material by releasing its internal stresses, similar to an annealing process (Fig. 6(b)-II).

The annealing effect also explains why the micro-hardness drops at the case of $100 \mathrm{~A}$ after the initial increases from the asreceived, to "Base line", and to E-ADSIF 50A (Fig. 6(c)). Note that the initial increase, was due to the repeated bending and unbending [18] at the local area. Interestingly, comparing the EADSIF to E-MDSIF, both performed at $50 \mathrm{~A}$, there exists a slightly positive increase of micro-hardness due to the two forming steps used in the MDSIF strategy.

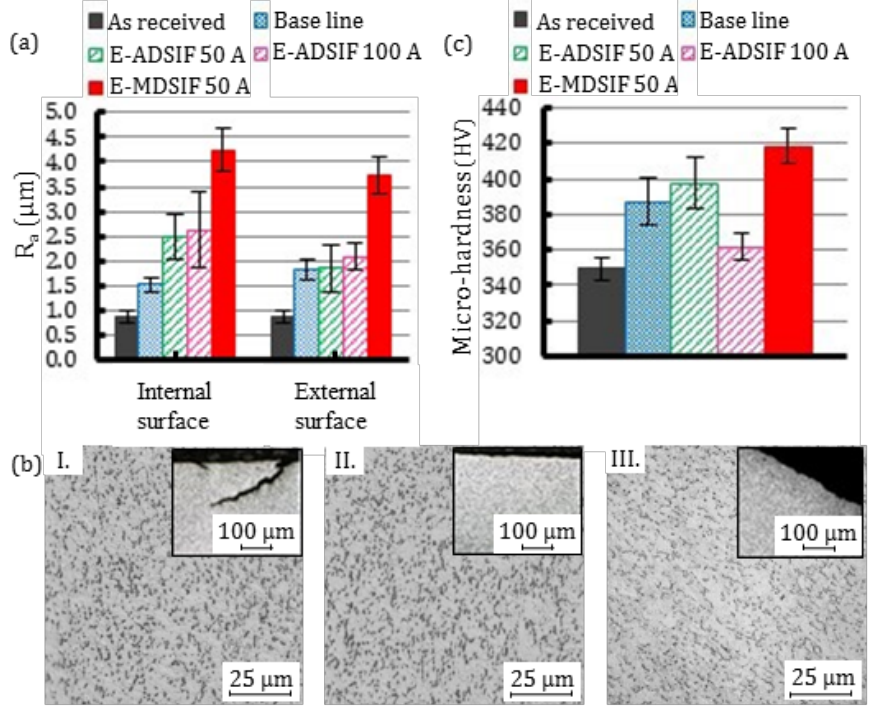

Figure 6. (a) Surface roughness, $\mathrm{R}_{\mathrm{a}}$ (b) Microstructure for I. base line, II. E-ADSIF 100 A, III. E-MDSIF 50 A samples. (c) Material micro-hardness as a function of forming process and current intensity.

\section{Conclusions}

E-MDSIF process, which applies electrical current with the mixed double-sided incremental forming (MDSIF) strategy, has been proved to be an effective strategy to manufacture patientspecific biomedical products using Ti6Al4V, improving the material formability and geometric accuracy. The methodology was applied successfully in the forming of a square pyramid part as well. The following conclusions were found:

1) The forming forces were reduced when the ADSIF process was electrically assisted: higher current intensities decreased the forming forces, though at 120 A non-desired thermal effects were observed.

2) E-MDSIF greatly enhanced the geometric accuracy compared to the ADSIF process performed with and without electricity.

3) The thermal softening from the Joule effect prevented surface defects from crack propagation that could bring an early fracture or future fatigue failure.

4) The E-MDSIF process did not affect the material microstructure and it proved to increase the material microhardness, as well as the surface roughness, which is related to the osseointegration of the implant.

5) The geometric accuracy and the high surface roughness enable E-MDSIF for the production of biomedical products.

In future work, the E-MDSIF will be applied using tool made in tungsten carbide (WC), which withstands higher temperatures. The aim is to increase the wall angle of the formed part and avoid the reduction of the geometric accuracy due to the wear of the steel tools at higher current intensities.

\section{Acknowledgments}

It is acknowledgeable the financial supports provided by the Spanish government (grant no. BES-2012-056760), University of Padova, and the U.S. Department of Energy (Award No. DEEE0005764).

\section{References}

[1] Valoppi, B., Ghiotti, A., Bruschi, S., Elevated temperature behaviour of Ti6Al4V sheets with thermo-electro-chemical modified surfaces for biomedical applications, Journal of Materials: Design and Application, DOI: 10.1177/1464420715601880.

[2] Ambrogio, G., De Napoli, L., Filice, L., Gagliardi, F., Muzzupappa, M., 2005, Application of incremental forming process for high customised medical product manufacturing, Journal of Materials Processing Technology, 162-163: 156-162.

[3] Xu, D.-K., Lu, B., Cao, T.-T., Zhang, H., Chen, J., Long, H., Cao, J., 2016, Enhancement of process capabilities in electrically-assisted double sided incremental forming, Materials and Design, 92: 268-280.

[4] Duflou, J.-R., Verbert, J., Belkassem, B., Gu, J., Sol, H., Henrard, C., Habraken, A.-M., 2008, Process window enhancement for single point incremental forming through multi-step toolpath, Annals of the CIRP, 57/ 1:253-256.

[5] Malhotra, R., Bhattacharya, A., Kumar, A., Reddy, N.-V., Cao, J., 2011, A new methodology for multi-pass single point incremental forming with mixed toolpaths, Annals of the CIRP, Vol.60/1: 323-326.

[6] Meier, H., Smukala, V., Dewald, O., Zhang, J., 2007, Two point incremental forming with two moving forming tools, Key Engineering Materials, 344: 599-605.

[7] Malhotra, R., Cao, J., Beltran, M., Xu, D., Magargee, J., Kiridena, V., Cedric Z.-X. 2012, Accumulative-DSIF strategy for enhancing process capabilities in incremental forming, Annals of the CIRP, 61/ 1: 251-254.

[8] Zhang, Z., Ren, H., Xu, R., Moser, N., Smith, J., Ndip-Agbor, E., Malhotra, R., Xia, J.-C., Ehmann, K.-F., Cao, J., 2015, A mixed double-sided incremental forming toolpath strategy for improved geometric accuracy, Journal of Manufacturing Science and Engineering, 137(5): 051007.

[9] Fan, G., Gao, L., 2014, Mechanical property of Ti-6Al-4V sheet in one-sided electric hot incremental forming, International Journal of Manufacturing technologies, 72: 989-994.

[10] Bagno, A., Di Bello, C., 2004, Surface treatments and roughness properties of Tibased biomaterials, Journal of Materials Science: Materials in Medicine, 15: 935-949.

[11] Li, Y., Zou, S., Wang, D., Feng, G., Bao, C., Hu, J., 2010, The effect of hydrofluoric acid treatment on titanium implant osseointegration in ovariectomized rats, Biomaterials, 31: 3266-3273.

[12] Meier, H., Magnus, C., 2013, Incremental sheet metal forming with direct resistance heating using two moving tools, Key Engineering Materials, 554-557: 1362-1367

[13] Moser, N., Zhang, Z., Ren, H., Zhang, H., Shi, Y., Ndip-Agbor, E., Lu, B., Chen, J., Ehmann, K.F., and Cao, J., 2016, Effective forming strategy for double-sided incremental forming considering in-plane curvature and tool direction, submitted to CIRP Annual.

[14] Vander Voort, G., Committee, A.I.H., 2004, ASM Handbook, ASM International. [15] Sánchez Egea, A.J., González Rojas, H.A., Celentano, D.J., Jorba Peiró, J., 2015, Mechanical and metallurgical changes on 308L wires drawn by electropulses. Materials and Design, 90:1159-1169.

[16] Ambrogio, G., Gagliardi, F., Bruschi, S., Filice, L., 2013, On the high-speed single point incremental forming on titanium alloys, Annals of the CIRP, 62/1: 243-246.

[17] Ambrogio, G., Filice, L., Gagliardi, F., 2012, Formability of lightweight alloys by hot incremental forming, Materials and Design, 34: 501-508.

[18] Smith, J., Malhotra, R., Liu, W.K., Cao, J., 2013, Deformation mechanism in singlepoint and accumulative double-sided incremental forming, International Journal of Manufacturing Technology, 69: 1185-1201. 